\title{
PROPOSTAS TERAPÊUTICAS PARA OS DESVIOS FONOLÓGICOS: DIFERENTES SOLUÇÕES PARA O MESMO PROBLEMA
}

\section{Treatment proposals for speech disorders: different solutions for the same problem}

\author{
Fernanda Marafiga Wiethan ${ }^{(1)}$, Helena Bolli Mota ${ }^{(2)}$
}

\begin{abstract}
RESUMO
Tema: enfoque das pesquisas realizadas nos últimos dez anos sobre terapia para os desvios fonológicos. Objetivo: realizar análise dos estudos em terapia fonológica, nacionais e internacionais, publicados a partir do ano de 2000 , a fim de verificar as contribuições destes para a clínica fonoaudiológica, além de apontar novas possibilidades em pesquisa. Conclusão: todas as pesquisas trazem contribuições que podem ser adotadas como recursos na clínica fonoaudiológica. No entanto, não foram encontrados estudos apontando o número médio de sessões necessárias para determinação da alta fonoaudiológica nos casos de desvio fonológico, bem como abordagens globais, que dessem conta de todos os aspectos que podem estar defasados nessas crianças, como processamento auditivo, memória de trabalho, discriminação fonêmica, alterações de consciência fonológica e desenvolvimento da linguagem escrita.
\end{abstract}

DESCRITORES: Fala; Distúrbios da Fala; Fonoterapia

\section{INTRODUÇÃO}

Desde a década de 1970, grande atenção tem sido dada à aquisição fonológica, típica ou atípica, pelas crianças. Os estudos em aquisição realizados até então, permitem ao clínico o conhecimento dos padrões normais e desviantes do desenvolvimento fonológico, auxiliando sobremaneira no diagnóstico do desvio fonológico (DF). A partir da determinação deste diagnóstico, torna-se extremamente importante a busca por estratégias que visem à adequação dos padrões de fala, levando a criança ao maior número de generalizações possível e reduzindo o tempo necessário para a supressão destas alterações.

(1) Fonoaudióloga, Mestranda em Distúrbios da Comunicação Humana pela Universidade Federal de Santa Maria (UFSM), Bolsista CAPES, Santa Maria, RS, Brasil.

(2) Fonoaudióloga, Doutora em Lingüística Aplicada pela Pontifícia Universidade Católica do Rio Grande do Sul. Professora Associada do Curso de Fonoaudiologia e do Programa de Pós-Graduação em Distúrbios da Comunicação Humana da Universidade Federal de Santa Maria (UFSM), Santa Maria, RS, Brasil.

Conflito de interesses: inexistente
Assim, os modelos de terapia fonológica foram criados para orientar o clínico na conduta a ser adotada para a resolução do DF. Os principais modelos utilizados na terapia são o Modelo de Ciclos ${ }^{1}$ e o Modelo de Ciclos Modificado2; os modelos com base em Pares Mínimos, em que se destaca o Modelo de Oposições Máximas3; o Modelo ABAB-Retirada e Provas Múltiplas ${ }^{4}$ e o Modelo Metaphon ${ }^{5}$. Mais recentemente tem-se o Modelo de Oposições Múltiplas ${ }^{6-7}$, que também tem base em pares mínimos.

No Brasil, estes modelos de terapia fonológica começaram a ser testados para falantes do português brasileiro na década de 1990, e vêm recebendo comparações e propostas de modificações desde então ${ }^{8-17}$.

A maior parte das pesquisas em terapia fonológica usa a generalização como critério de determinação do sucesso terapêutico. A generalização é abordada nos trabalhos que envolvem terapia fonológica, por ser o principal meio de se verificar a evolução do Sistema Fonológico (SF) 13-15,18-21.

A partir dos avanços nos estudos sobre o DF e a terapêutica para estes casos, o volume de trabalhos 
abordando este tema cresceu consideravelmente na última década, ampliando as possibilidades de escolha por parte dos clínicos. Assim, alguns autores "ousam" ao trazer modelos ou técnicas terapêuticas diferenciadas ou propõem modificações aos modelos existentes, outros realizam estudos comparando abordagens terapêuticas já consagradas a fim de verificar a mais efetiva para cada caso ou, pesquisas buscam a solução do DF a partir de atividades de consciência fonológica (CF). Ainda, há alguns trabalhos que investigam a influência do controle sobre os sons ou palavrasalvo escolhidas para o tratamento na ocorrência de generalizações e redução do tempo de terapia.

Verifica-se assim, que existem inúmeros estudos sobre o assunto, tomando diferentes enfoques, 0 que possibilita a seleção das pesquisas mais bemsucedidas para aplicação na clínica fonoaudiológica, além do conhecimento dos aspectos pouco ou não abordados.

Deste modo, o objetivo desta revisão de literatura é realizar a análise dos estudos em terapia fonológica, nacionais e internacionais, publicados a partir do ano de 2000 , a fim de verificar as contribuições destes para a clínica fonoaudiológica, além de apontar novas possibilidades em pesquisa.

\section{MÉTODO}

As ferramentas de busca para esta revisão de literatura foram o Medline e o Lilacs por intermédio da pesquisa avançada simultânea de base de dados da biblioteca virtual em saúde da Bireme, além de consulta aos Periódicos CAPES no período de novembro de 2009 a fevereiro de 2010. Buscouse por artigos originais de pesquisa e relatos de casos clínicos.

Além da bibliografia obtida por meio das bases de dados, foram utilizados livros, monografias, dissertações, teses e artigos de periódicos impressos.

As palavras-chave utilizadas para a busca foram: "Fala e fonoterapia", "Distúrbios da Fala", "Fonoterapia" e "Terapia Fonológica", além dos termos em inglês: "Speech Disorders", "Speech Therapy", "Phonological approach".

Priorizou-se utilizar referências publicadas na última década, exceto quando se tratavam de trabaIhos clássicos e/ou de extrema relevância para o tema desta pesquisa. Realizou-se pré-seleção do material estudado a partir da leitura dos resumos e, após esta pré-seleção, todos os trabalhos foram lidos na íntegra. Na pré-seleção foram descartados trabalhos relacionados a alterações de fala que não fossem de ordem fonológica ou trabalhos que não fossem diretamente relacionados ao tema da pesquisa.

\section{REVISÃO DA LITERATURA}

Destaca-se que os artigos que serão apresentados a seguir não se encontram dispostos em ordem cronológica, mas sim agrupados conforme o assunto abordado, a fim de facilitar a exploração do assunto. Foram utilizados 48 trabalhos, dos quais, 39 artigos, sendo 20 provenientes de periódicos nacionais e 19 de periódicos internacionais; três livros escritos em língua inglesa; três teses e três dissertações, todas brasileiras.

\section{Abordagens Diferenciadas}

Além das mudanças a que os modelos de terapia fonológica são submetidos, alguns autores sugerem novas propostas, diferenciadas das que levam em conta exclusivamente o nível fonológico e/ou articulatório da linguagem.

Neste sentido, a abordagem psicolingüística visa compreender de que forma o modelo de processamento da linguagem da criança está deficitário, e determinar a melhor maneira para sanar esta dificuldade. Além disso, de acordo com esta abordagem, cada indivíduo deve ser considerado único e, por isso, não é possível generalizar um determinado tipo de terapia para qualquer que seja a alteração de fala e/ou linguagem apresentada. Um estudo de caso com um sujeito apresentando DF de grau grave e persistente evidenciou que a abordagem psicolinguística foi eficaz, pois a paciente obteve mudanças significantes na fala e em outras áreas, como soletração e discriminação auditiva, sendo que as mudanças foram mantidas meses após o término da intervenção 22 .

A abordagem centrada no vocabulário tem sido utilizada para casos de DF que apresentam padrões de erros de fala incomuns e inconsistentes. Esta abordagem visa ensinar à criança como organizar os fonemas na palavra, primeiramente em palavras isoladas e após, em fala encadeada. São utilizados vocábulos que sejam parte do vocabulário funcional das crianças. Não há preocupação com a extensão das palavras ou com a complexidade fonêmica. Também não há fase de imitação do terapeuta, como ocorre em outros modelos, e a produção da criança é aceita mesmo se for incorreta, desde que os erros sejam compatíveis com o padrão de desenvolvimento fonológico. Portanto, o objetivo do modelo é transformar os processos fonológicos idiossincráticos em erros comuns e consistentes ${ }^{23}$.

Utilizando a abordagem descrita anteriormente ${ }^{23}$, foi realizado um estudo de caso sobre o tratamento de três meninos com DF, apresentando processos fonológicos idiossincráticos e inconsistentes com fala ininteligível. Todas as crianças já haviam sido submetidas à terapia fonoaudiológica, 
sem obter sucesso. As sessões ocorriam duas vezes por semana e tinham duração entre 30 e 40 minutos. O número total de sessões variou entre os sujeitos: 12, 27 e 38. Ao final do tratamento, todos os meninos evidenciaram ganhos em inteligibilidade, precisão e consistência na produção das palavras. A abordagem centrada no vocabulário mostrou ser apropriada para todas as crianças, embora tenha havido diferenças individuais ${ }^{24}$.

A abordagem PACT (parents and children together) envolve a participação dos cuidadores na terapia de crianças com DF. Os componentes desta abordagem são: educação dos pais; treino metalinguístico; treino da produção fonética; terapia com pares mínimos e bombardeio auditivo; além de tarefas para casa. Este tipo de terapia é desenvolvido com blocos de terapia e retirada, durante a qual os pais trabalham com as crianças em casa ${ }^{25}$. Os criadores desta abordagem apresentaram o caso clínico de um menino de sete anos de idade, com DF grave e fala essencialmente ininteligível, em que foi utilizado este modelo. Os autores encontraram dificuldades para aplicar a terapia PACT, especialmente pelo fato desta ser mais apropriada para crianças de três a seis anos de idade ${ }^{26}$.

Outros autores buscaram investigar a eficácia de uma abordagem eclética para o tratamento dos DF, submetendo as crianças à terapia mais coerente com a prática clínica e à abordagem que conta com a participação dos pais no tratamento de seus filhos. Conforme o estudo, os clínicos em geral, costumam utilizar-se de diferentes abordagens simultaneamente, a fim de promover mudanças nos SF de seus pacientes, porém isto não é utilizado nas pesquisas científicas. Assim, os autores realizaram dois pequenos experimentos em clínicas de Fonoaudiologia. No primeiro, o grupo de crianças tratadas foi comparado com um grupo sem tratamento direto, no qual foi utilizada apenas a intervenção dos pais por meio de tarefas para serem executadas em casa. O segundo comparou as crianças tratadas em casa pelos pais com crianças que não foram submetidas a tratamento algum. Verificou-se então que: no primeiro experimento todas as crianças demonstraram avanços estatisticamente significantes. No segundo experimento, as crianças tratadas obtiveram ganhos significativos, enquanto as não tratadas não demonstraram mudanças. Ressalta-se que as crianças tratadas pelos terapeutas tiveram significativamente mais ganhos do que as tratadas pelos pais. Conclui-se deste modo que, tanto a abordagem eclética quanto a abordagem que envolve os pais na terapia são eficientes no tratamento do DF ${ }^{27}$. Verificar a eficácia do trabalho realizado pelos fonoaudiólogos em clínicas ou consultórios é de extrema relevância, visto que os recursos utilizados por estes parecem ser diferentes do que é trabalhado no meio científico. Seria interessante ainda, comparar o trabalho destes profissionais com a aplicação dos tradicionais modelos de terapia com base fonológica, que são os mais utilizados em meio acadêmico.

A abordagem psicolinguística traz uma valiosa contribuição para a clínica fonoaudiológica, em geral, ao enfatizar que cada indivíduo é único e, por isso, necessita de um planejamento específico para seu caso. Percebe-se que, muitas vezes, é escolhida uma única abordagem para ser aplicada a todos os sujeitos que apresentam determinada patologia, sem ser considerada a individualidade e as características de cada paciente, o que pode comprometer negativamente o processo terapêutico.

A abordagem centrada no vocabulário parece ser importante para as crianças que apresentam processos fonológicos idiossincráticos e inconsistentes em sua fala, o que tem sido preocupação frequente no meio científico. Porém, à medida que os erros de fala tornam-se consistentes, seria mais apropriado utilizar uma abordagem ou estratégia terapêutica que vise à produção correta para a obtenção de ganhos mais significantes.

\section{Comparação entre abordagens terapêuticas}

Existem inúmeros artigos comparando abordagens terapêuticas na literatura científica. Verificase que a maioria dos artigos brasileiros explora os modelos ABAB-Retirada e Provas Múltiplas, Ciclos e Ciclos Modificado e os modelos de Pares Mínimos. Já os trabalhos internacionais são mais ecléticos na aplicação e comparação das abordagens terapêuticas. Seja qual for a comparação realizada, estes estudos são relevantes à medida que podem determinar a superioridade de determinado procedimento sobre outro, conduzindo o clínico à conduta terapêutica que trará mais ganhos ao paciente.

A fim de comparar a abordagem metafonológica com a abordagem articulatória no tratamento de crianças com DF, foram pesquisadas 61 crianças, com idades entre três anos e seis meses e cinco anos. O estudo contou com um grupo controle formado por 59 crianças com desenvolvimento fonológico normal, na mesma faixa etária estudada, a fim de controlar os efeitos maturacionais. Foram realizadas avaliações de fala e CF antes e após a intervenção terapêutica para todos os sujeitos e três meses após a terapia para as crianças com DF. Os sujeitos submetidos a ambas as abordagens terapêuticas evidenciaram evolução estatisticamente significante tanto na fala quanto em CF, quando comparadas às crianças do grupo controle. Porém não houve significância estatística entre as 
abordagens terapêuticas estudadas. Os grupos diferiram apenas em relação à melhora da fala, em que o grupo submetido à terapia articulatória obteve mais mudanças. A avaliação realizada três meses após a intervenção evidenciou que, houve uma tendência para o grupo submetido à terapia metafonológica continuar mostrando mais mudanças a longo prazo ${ }^{28}$.

Outro estudo internacional comparou as abordagens: contrastiva, abordagem centrada no vocabulário (Core Vocabulary) e PROMPT (Prompts for Restructuring Oral Muscular Phonetic Targets phonological contrast). Este último modelo terapêutico enfoca o controle e a programação dos movimentos motores, utilizando fonemas isolados e vocábulos a partir de pistas táteis. As três crianças foram submetidas aos três modelos, sendo que a terapia, portanto, constou de três etapas (um modelo em cada etapa). Todos os modelos demonstraram ser responsáveis por melhora significativa da inteligibilidade de fala das crianças. A intervenção baseada no contraste de pares mínimos mostrou-se mais efetiva para a criança que apresentava estratégias de reparo comuns do desenvolvimento fonológico. Já as crianças que apresentavam estratégias de reparo inconsistentes, se beneficiaram mais com a abordagem centrada no vocabulário. Entretanto, assim que os erros tornavam-se comuns, uma criança passou a se beneficiar mais com a terapia baseada nos contrastes de pares mínimos. A abordagem PROMPT foi a que menos evidenciou mudanças nos SF dos três sujeitos. Os resultados sugerem que, as respostas às abordagens terapêuticas variam de acordo com o tipo de erro fonológico apresentado por cada criança, portanto, não existe um modelo que possa ser utilizado para todos os sujeitos com $\mathrm{DF}^{29}$.

Resultados semelhantes foram encontrados em pesquisa que comparou a abordagem contrastiva à abordagem centrada no vocabulário: todas as crianças do estudo melhoraram a inteligibilidade de fala e, a terapia centrada no vocabulário resultou em maiores mudanças para as crianças com padrões de erros de fala inconsistentes e a abordagem contrastiva proporcionou mais mudanças para as crianças com estratégias de reparo comuns do desenvolvimento ${ }^{30}$.

Conforme observado, a abordagem centrada no vocabulário parece ser efetiva apenas até o momento em que a criança torna seus erros de fala consistentes. Ainda, novamente foi mostrada a importância de considerar as diferenças individuais na escolha do tratamento.

Uma pesquisa, publicada em 2007, objetivou verificar a generalização a itens não utilizados no tratamento obtida em três modelos de terapia
(Modelo de Ciclos Modificado, Modelo ABAB-Retirada e Provas Múltiplas, e Modelo de Oposições Máximas Modificado) em sujeitos com diferentes graus de gravidade do DF. Os resultados evidenciaram que, dos sujeitos com desvio grave, moderado-grave e médio, três apresentaram maiores generalizações a outras palavras, todos submetidos ao Modelo ABAB-Retirada e Provas Múltiplas. Dos sujeitos com desvio médio-moderado, foram os tratados pelo Modelo de Oposições Máximas Modificado que mais generalizaram ${ }^{13}$. As mesmas autoras publicaram outro estudo em 2008, comparando os mesmos modelos, porém utilizando como critério, todos os tipos de generalização estrutural possíveis. Os resultados obtidos evidenciaram novamente, que a generalização dentro de uma classe de sons ocorreu em todos os modelos pesquisados, além de observar-se certa "desvantagem" do Modelo de Ciclos Modificado nos diferentes aspectos avaliados ${ }^{14}$. No ano de 2009 as autoras publicaram os dados relacionados à generalização para outra posição na palavra encontrando os mesmos resultados descritos anteriormente $^{15}$. Nas três pesquisas, é importante atentar para alguns fatores: embora as amostras tenham sido expressivas, não houve tratamento estatístico dos dados analisados e não foi considerado o número de sessões a que cada sujeito foi submetido, o que deve ser determinante na predição do sucesso terapêutico.

Ainda comparando estes três modelos, porém utilizando-se de análise estatística, foram encontradas algumas diferenças em relação aos três estudos anteriores. Os elementos para comparação foram as mudanças referentes ao SF, com base na comparação do número de fonemas adquiridos, número de sons estabelecidos no inventário fonético e traços distintivos alterados, antes e após a terapia. Observou-se diferença estatisticamente significante entre as avaliações iniciais e finais nos três modelos quanto aos fonemas estabelecidos no SF e traços distintivos alterados. Não houve diferença estatística entre os modelos terapêuticos, concluindo-se deste modo que todos foram efetivos de modo semelhante no tratamento de crianças com DF ${ }^{16}$.

Avaliando as mudanças fonológicas ocorridas no SF de 66 sujeitos com diferentes gravidades do DF, outra pesquisa também comparou estes três modelos, avaliando o SF antes após um período de 15 a 25 sessões de terapia. Em todos os grupos estudados houve aumento do percentual de consoantes corretas (PCC) e do percentual de sons adquiridos, sendo que estes resultados foram estatisticamente significativos para a maioria dos grupos. 
Além disso, as maiores mudanças no SF ocorreram para os grupos com DF de grau mais acentuado ${ }^{31}$.

Os modelos tradicionais de terapia fonológica, são eficientes, na mesma proporção e, por isso, continuam sendo utilizados e pesquisados.

Um software foi desenvolvido para terapia dos DF, envolvendo jogos para incentivar a produção correta das palavras-alvo. As autoras compararam três grupos por meio de avaliações pré e pós-terapia. O primeiro grupo era composto por crianças que receberam a intervenção por meio do software, o segundo grupo recebeu terapia convencional e o terceiro grupo não recebeu intervenção. Os resultados mostraram que não houve diferença significativa entre os grupos, nem mesmo em relação ao grupo controle. Os autores atribuem este resultado à quantidade de sessões e à frequência da terapia, além da heterogeneidade entre os grupos. A estimulabilidade para os sons-alvo e o gênero das crianças foram também considerados causas possíveis para os resultados apresentados. Assim, as autoras sugerem que estudo similar seja realizado para comparar os métodos de terapia, utilizando grupos mais homogêneos em termos de estimulabilidade, além de realizar um maior número de sessões de intervenção ${ }^{32}$. Observa-se a necessidade de criação e utilização de softwares que auxiliem as terapias de fala. Na literatura são encontrados muitos recursos como estes para outras áreas da fonoaudiologia, mas poucos, especificamente para terapia fonológica.

Outro estudo objetivou comparar a abordagem de pares mínimos - oposições mínimas à abordagem de pares mínimos - oposições máximas. Foram tratadas 19 crianças, sendo nove com oposições mínimas e 10 com oposições máximas. Os resultados do estudo revelaram que todas as crianças demonstraram evolução significativa após seis sessões de terapia, porém não houve diferenças entre os dois grupos ${ }^{33}$. Também comparando estes dois modelos, outro estudo comparou 25 sessões de terapia de dois irmãos, com diferentes idades e gravidades do DF, sendo um (S1) tratado pelo Modelo de Oposições Mínimas e outro (S2) pelo Modelo de Oposições Máximas. Os resultados evidenciaram que a criança tratada por este último modelo apresentou melhor evolução terapêutica, porém é importante ressaltar que S2 apresentava grau do desvio moderado-grave e S1, grave. As autoras concluíram que ambos os modelos foram eficazes por proporcionarem melhora no SF de ambas as crianças a partir das generalizações ${ }^{34}$.

\section{Atividades de Consciência Fonológica na Terapia Fonológica}

Embora se saiba que a maioria das crianças portadoras de DF apresenta déficits de CF, atividades desta natureza ainda não estão sendo amplamente utilizadas na prática clínica. Este fato pode ser confirmado a partir dos resultados de um estudo que concluiu que, mesmo após a determinação da alta fonoaudiológica, os sujeitos que eram portadores de DF, apresentaram déficits na maioria das tarefas de $\mathrm{CF}^{35}$. Desta forma, é importante ressaltar estudos que tragam esta temática.

A fim de pesquisar a hipótese descrita anteriormente, foi realizado um estudo com o objetivo de verificar as habilidades de fala, escrita e linguagem de crianças que haviam sido submetidas à terapia fonológica e metafonológica. Quando os sujeitos do estudo freqüentavam a pré-escola e apresentavam DF, foram submetidos a um programa de terapia fonológica baseado na fonologia não-linear, e metafonológica com tarefas de rima e aliteração. Todas as crianças obtiveram ganhos significativos em fonologia e, algumas em CF. Três anos após o término desta intervenção, as autoras investigaram as habilidades de fala, escrita e linguagem destas crianças, verificando que: das doze crianças da amostra, cinco apresentavam erros residuais de fala; somente duas crianças mostraram habilidades abaixo da média para leitura (decodificação e compreensão), embora cinco tenham apresentado médias abaixo do esperado em soletração; crianças que apresentavam limitações em memória verbal, produção linguística e CF evidenciaram mais déficits no processo de alfabetização; as crianças com melhor desenvolvimento da escrita foram as que tiveram melhor desempenho nas tarefas de CF durante o programa de intervenção. Assim, as autoras concluíram que, a intervenção precoce em fonologia e CF pode promover normalização do desenvolvimento da fala e da aquisição das habilidades de leitura e escrita ${ }^{36}$.

A fim de examinar a eficácia da terapia de CF para a melhora das habilidades de fala e escrita de crianças com DF, uma pesquisa avaliou o tratamento de dez crianças, comparando-o com o grupo controle. Os sujeitos foram tratados em grupos de três crianças cada, totalizando 12 sessões de terapia. Ao final do estudo, o grupo tratado evidenciou ganhos significativos em CF. Entretanto, diferenças entre os grupos nos aspectos de fala e escrita foram menores e não mostraram significância estatística. Os resultados sugerem que, embora a terapia tenha sido efetiva para melhorar as habilidades de CF, um maior número de sessões seria necessário para se observar evolução dos 
aspectos de fala e escrita, sendo necessárias mais pesquisas para confirmar estas hipóteses ${ }^{37}$.

Outro estudo teve o objetivo de investigar a possibilidade de ensinar habilidades de consciência fonêmica para crianças em fase de pré-alfabetização que apresentavam DF. A amostra constou de 42 crianças com idades entre quatro anos e quatro anos e seis meses, todas apresentando DF. Para a metade das crianças, foi aplicado um programa envolvendo aliteração, segmentação, manipulação e síntese fonêmica e para a outra metade, foi aplicado um programa de estimulação da linguagem. De modo geral, as autoras verificaram que o grupo tratado com atividades de consciência fonêmica mostrou mais avanços (com significância estatística) do que o grupo tratado com estimulação da linguagem. A conclusão do estudo mostra que é possível ensinar tarefas de consciência fonêmica para as crianças em fase de pré-alfabetização, porém as atividades de manipulação e segmentação são mais difíceis, pois apenas as crianças com melhor desenvolvimento cognitivo e mais velhas conseguiram obter sucesso nestas tarefas. Além disso, todas as crianças do estudo aprimoraram a fala ${ }^{38}$.

Aplicando-se um programa de atividades estimulando as habilidades de $\mathrm{CF}$, tendo como base teórica a hierarquia dos traços distintivos, foram tratadas quatro crianças com DF de seis a oito anos de idade. O processo terapêutico teve duração de 13 sessões de 30 minutos cada. Os resultados mostraram que todos os pacientes apresentaram evolução significativa após o término do programa, superando todos os processos fonológicos presentes e adquirindo os traços que estavam ausentes. Os pacientes ampliaram seu SF à medida que melhoraram seu desempenho nas atividades de $\mathrm{CF}^{39}$.

\section{Cuidados na seleção dos alvos de tratamento}

Esta temática pode ser analisada sob diversos prismas, pois se pode explorar a escolha dos sonsalvo ou das palavras-alvo, além disso, pode ser considerado o contexto linguístico de determinado som-alvo ou o quanto este é estimulável, ou seja, a capacidade de repetição do fonema pela criança. Alguns autores, ainda propõem listas de palavras possíveis de serem utilizadas em terapia. Deste modo, atualmente são frequentes os estudos sobre este tema no Brasil.

Em 2001 foi proposta uma lista de palavras-alvo que podem ser utilizadas no tratamento de crianças com DF a partir da abordagem de pares mínimos. A autora propõe uma lista com todos os possíveis contrastes de fonemas consonantais do Português, classificando-os quanto ao número de traços diferentes entre fonemas e o tipo de distinção. Sugere ainda, exemplos de pares mínimos para cada contraste ${ }^{40}$. Trabalhos como este são extremamente importantes, especialmente pela possibilidade de serem utilizados didaticamente para alunos de Fonoaudiologia que estejam iniciando no atendimento a pacientes, por apresentarem pouca prática no planejamento terapêutico e execução do mesmo, ou ainda, para o clínico que não conhece e deseja utilizar os modelos com base fonológica.

Quanto à estimulabilidade do som-alvo, uma pesquisa objetivou verificar o desempenho nas produções corretas de três sujeitos com DF, antes e após um ciclo de tratamento, quando tratados com fonemas-alvo estimuláveis e não estimuláveis, utilizando o Modelo ABAB-Retirada e Provas Múltiplas. Participaram do estudo três sujeitos: um (S1), tratado com um fonema-alvo estimulável, e outros dois sujeitos (S2 e S3), que foram tratados com fonemas-alvo não estimuláveis. Observou-se que o fonema-alvo estimulável provocou maiores ganhos no SF de S1 quando comparado às modificações provocadas pelos fonemas-alvo não estimuláveis nos SF de S2 e S3. Entretanto, as autoras colocam que novos estudos sobre as estratégias de escolha dos fonemas-alvo são necessários, com uma amostra mais expressiva e maior número de sessões ${ }^{41}$.

Especificamente, em relação à seleção das palavras-alvo para terapia, os ambientes favoráveis consistem de contextos facilitadores à produção e aquisição do segmento tratado ${ }^{42}$. Alguns autores s2-46 $^{42}$ estudaram a relevância destes contextos na facilitação da produção correta e aquisição fonêmica por parte das crianças.

Uma autora realizou um levantamento das pesquisas sobre a aquisição do fonema /r/ sugerindo, a partir destes estudos, uma hierarquia de palavras em relação ao padrão de facilitação linguística. Estes padrões de facilitação foram divididos em 12 níveis hierárquicos, considerando a posição que o segmento ocupa na sílaba e na palavra, o ambiente fonético antecedente e seguinte, a tonicidade da sílaba em que o segmento está inserido e o número de sílabas das palavras. Assim, as palavras mais favorecedoras (padrão 1) foram "peru" e "peruca", devido ao /r/ estar antecedido de /i/ (realização fonética) e na sílaba tônica. As menos favorecedoras estão no padrão 10, "zero" e "pérola", são antecedidas pela vogal /ع/ e estão em sílaba postônica ${ }^{43}$.

A partir dos pressupostos teóricos da pesquisa descrita anteriormente ${ }^{43}$, foi realizada uma análise das palavras-alvo selecionadas para terapia, no estudo de caso de um menino, apresentando DF, com seis anos e oito meses. Foi aplicado o modelo 
ABAB- Retirada e Provas Múltiplas, utilizando-se todas as palavras na posição de onset medial, a posição mais favorável; apenas três palavras com /r/ na sílaba tônica (favorável) e nenhuma das palavras encontrava-se em ambiente favorável quanto ao contexto antecedente e seguinte. Os resultados indicaram que as palavras que continham ambientes facilitadores foram produzidas corretamente um maior número de vezes durante o tratamento. As autoras sugerem, que mais estudos sejam realizados analisando ambientes favoráveis no tratamento para outros modelos, como Modelo de Ciclos ou Oposições Máximas ${ }^{42}$.

Em 2009, novo estudo foi realizado utilizando os mesmos pressupostos teóricos ${ }^{43}$ objetivando analisar a aquisição de /r/ em duas crianças com DF, comparando-as por meio da generalização obtida. Foram considerados os ambientes tonicidade da sílaba, contexto precedente e contexto seguinte da líquida não-lateral na posição de onset medial. Um sujeito foi submetido à terapia com os sons-alvo em contextos mais favoráveis e o outro em contextos menos favoráveis utilizando-se para ambos o modelo ABAB-Retirada e Provas Múltiplas. Os resultados evidenciaram melhor evolução terapêutica no sujeito tratado com o contexto mais favorável, demonstrando que a escolha das palavras-alvo para terapia baseando-se em variáveis lingüísticas facilitadoras é um fator importante para a aquisição fonológica e para a ocorrência de generalizações ${ }^{46}$.

Uma dissertação de mestrado também foi dedicada à pesquisa sobre o papel dos contextos facilitadores na aquisição de /r/ por crianças com DF. A autora em questão comparou o tratamento, por meio do modelo ABAB-Retirada e Provas Múltiplas, de sete crianças a partir das abordagens gerativa e gestual, testando ambientes neutros e ambientes favoráveis. Todas as crianças possuíam grau de desvio médio e dificuldades apenas na produção do fonema $/ r /$. Os resultados não indicaram diferenças entre as abordagens gerativa e gestual, porém o contexto neutro mostrou-se mais favorecedor para aquisição do segmento, na posição de onset medial e coda medial do que o contexto favorável. Uma das conclusões do estudo foi que o contexto lingüístico favorável de ambas as abordagens teóricas consideradas (gerativa e gestual) não é aplicável à seleção das palavras no tratamento do DF. Porém, é necessário considerar que há divergências na literatura em relação à quais contextos são favoráveis ou neutros para aquisição do fonema $/ r / 44$.

Outra pesquisa encontrada sobre a aplicabilidade dos ambientes favoráveis em terapia é uma tese de doutorado. Na primeira etapa da pesquisa, a autora pesquisou a aquisição fonológica dos segmentos /f/, /v/, / / / e /3/ de 46 crianças com desenvolvimento fonológico atípico, na segunda etapa, oito crianças desta amostra foram selecionadas e tiveram seu processo terapêutico analisado. Nesta última etapa, o objetivo foi verificar o efeito do valor de favorecimento que as palavrasalvo de tratamento, gerado pela tonicidade, número de sílabas, posição na palavra, contexto fonológico precedente e seguinte, envolvendo as fricativas, tem no sucesso da terapia. O modelo de terapia, da mesma forma que nas outras pesquisas, foi - ABAB-Retirada e Provas Múltiplas. Os dados da pesquisa foram submetidos à análise pelo programa computacional VARBRUL. Desta forma, a partir dos resultados selecionados pelo programa referentes aos valores de favorecimento absolutos, a autora atribuiu para cada valor um conceito, tendo cada conceito um peso que variou de 0 a 3 , considerando-se então a soma dos pesos para determinar o favorecimento da palavra. O resultado da soma indica se a palavra é muito favorável (peso 15), favorável (peso de 14 a 10), neutra (peso 9), pouco favorável (peso 8 a 3) ou desfavorável (peso igual ou inferior a 2). As conclusões obtidas a partir do estudo foram que: embora não haja uma relação diretamente proporcional entre as palavras favoráveis usadas em terapia e o número de sons adquiridos, os sujeitos que tiveram apenas uma ou nenhuma palavra-alvo favorável no tratamento foram os que menos apresentaram generalizações; as palavras-alvo selecionadas para serem usadas na terapia representam um papel favorecedor, porém não determinante na reorganização dos SF dos sujeitos com $\mathrm{DF}^{45}$.

Os estudos sobre ambientes linguísticos facilitadores ainda são pouco explorados e apresentam resultados discrepantes entre as pesquisas, sendo necessários mais estudos, com mais sujeitos, aplicando-se diferentes modelos de terapia fonológica, e utilizando também outras classes de sons, como as plosivas, por exemplo.

\section{Modificações propostas aos Modelos de Terapia Fonológica}

A partir da vivência dos modelos de terapia na prática clínica, muitos pesquisadores verificam a necessidade de modificar os modelos existentes ou inserir procedimentos que venham a contribuir para a evolução terapêutica adequada.

Uma das autoras do Modelo de Ciclos, ao apresentar o caso clínico de uma criança com DF, propõe a inserção de atividades de CF ao modelo, além de admitir maior flexibilidade na determinação do número de sessões semanais necessário e ampliação do número de palavras lidas para a criança no bombardeio auditivo ${ }^{47}$. 
O Modelo de Oposições Máximas foi modificado quanto à seleção dos alvos de tratamento, procedimentos terapêuticos e estrutura da sessão. Quanto ao primeiro item, faz-se necessária a realização de uma avaliação fonológica detalhada do SF da criança e uma análise por traços distintivos. O intuito destas avaliações é selecionar fonemas com os traços distintivos que o sujeito tenha dificuldade, atentando também neste momento para que as palavras-alvo tenham significado e, na medida do possível, ambientes fonéticos controlados. Em relação aos procedimentos terapêuticos, é realizada a linha de base a cada cinco sessões de estimulação do som-alvo até alcançar de 20 a 25 sessões. Quanto à estrutura da sessão, foi inserido o Bombardeio auditivo e a criança deve atingir $80 \%$ de produções corretas na fase de imitação para chegar à produção espontânea. Este modelo foi testado, em falantes do português brasileiro em um menino com DF de seis anos e quatro meses, mostrando-se eficaz em relação à melhora na inteligibilidade da fala, propiciando ampliação do inventário fonético da criança por meio da ocorrência de generalizações ${ }^{11}$.

Utilizando o modelo citado anteriormente, foi realizado um trabalho, cujo objetivo foi verificar as mudanças fonológicas obtidas pelo tratamento utilizando a abordagem do 'reforço' ou 'contraste', em sujeitos com diferentes gravidades do DF. A abordagem do 'contraste' utiliza dois sons-alvo que possuem valores opostos para o mesmo traço (mais marcado $X$ menos marcado), enquanto a do reforço considera o uso de sons-alvo com valores idênticos para o mesmo traço. Assim, três sujeitos com idade média de seis anos foram submetidos à terapia fonológica pelo modelo de Oposições Máximas Modificado, considerando-se a abordagem do 'reforço' ou 'contraste', verificando-se assim, as seguintes generalizações: para itens não utilizados no tratamento; para outra posição na palavra; dentro de uma mesma classe de sons; e para outra classe de sons. O inventário fonético dos três sujeitos estava completo após a terapia; porém, o SF ainda encontrava-se incompleto para todos os sujeitos. Após o processo terapêutico, observouse evolução para todos os tipos de generalização estrutural analisados, de modo equilibrado entre os sujeitos ${ }^{48}$.

O Modelo de Oposições Múltiplas ${ }^{6}$ recebeu uma proposta de modificação no tratamento de um sujeito do sexo masculino, falante do português brasileiro, com DF de grau moderado-grave e idade de 6:6. A modificação consistiu em considerar os traços distintivos alterados na escolha dos sonsalvo e na verificação das mudanças proporcionadas pela terapia. Os traços que sofriam maior número de alterações foram determinantes para a escolha dos sons-alvo /r/ x /// x /// x / 3/ x /z/, na posição de onset medial. Além disso, as autoras optaram por realizar pequenas modificações na estrutura da sessão terapêutica. Os resultados mostraram que após dez sessões de fonoterapia, o sujeito adquiriu os fonemas trabalhados, exceto $0 / \mathrm{r} /$, generalizando-os para outras posições na palavra, para a mesma classe de sons e para outras classes de sons. As autoras concluíram que a proposta mostrou-se efetiva, evidenciada pela melhora no SF da criança ${ }^{17}$.

É importante ressaltar os estudos que propõem modificações aos modelos terapêuticos existentes, já que a vivência com os modelos é que mostra suas falhas e as possibilidades de mudança. Deste modo, o terapeuta deve ter liberdade para modificar o modelo adotado de acordo com suas experiências anteriores e, principalmente, com as necessidades de cada paciente.

\section{CONCLUSÃO}

A partir dos trabalhos apresentados pode-se concluir que, apesar do grande volume de estudos na área de terapia fonológica, muito ainda pode ser feito com o objetivo de reduzir o tempo de terapia fonoaudiológica para crianças com DF, conforme foi sugerido. Além disso, todas as pesquisas trazem contribuições que podem ser adotadas como recursos na clínica fonoaudiológica.

Não foram encontrados estudos apontando o número médio de sessões necessárias para determinação da alta fonoaudiológica nos casos de desvio fonológico, bem como abordagens globais, que dessem conta de todos os aspectos que podem estar defasados nessas crianças, como processamento auditivo, memória de trabalho, discriminação fonêmica, alterações de consciência fonológica e desenvolvimento da linguagem escrita.

Ressalta-se a preocupação da maioria dos autores com as crianças que apresentam DF grave e/ou com fala ininteligível e processos fonológicos inconsistentes e idiossincráticos. As soluções apontadas para estes casos parecem estar sendo efetivas, embora ainda seja necessária a busca constante por novos recursos.

Por fim, salienta-se que o terapeuta deve atentar para o fato que crianças diferentes respondem de maneira diferenciada às diversas abordagens apresentadas, sendo necessária cautela por parte do clínico no planejamento terapêutico. 


\section{ABSTRACT}

Background: focus on studies conducted over the last ten years on therapy for phonological disorders. Purpose: to analyze the national and international studies on phonology, conducted from the year 2000 , in order to check their contributions for the clinical treatments, while identifying new opportunities in research. Conclusion: all analyzed studies bring out contributions that can be adopted as tools for the speech clinic. The therapist should pay attention to the fact that different children respond differently to the different approaches, so that therefore, caution should be taken clinician in treatment planning. The report suggests research possibilities and aspects that could be more widely used in the scientific work. However, no studies were found indicating the average number of sessions required in order to determine the discharge in speech pathology cases, and global approaches, that could solve all the issues that may be delayed in these children, such as auditory processing, working memory, phonemic discrimination, phonological awareness and written language development.

KEYWORDS: Speech; Speech Disorders; Speech Therapy

\section{REFERÊNCIAS}

1. Hodson BW, Paden EP. Targeting intelligible speech: A phonological approach to remediation. San Diego, CA: College Hill Press, 1983.

2. Tyler A, Edwards ML, Saxman J. Clinical application of two phonologically based treatment procedures. J Speech Hear Disorders. 1987; 52: 393-409.

3. Gierut JA. Maximal opposition approach to phonological treatment. J. Speech Hear. Dis.1989; 54: 9-19.

4. Tyler A, Figursky GR. Phonetic inventory changes after treating distinctions along an implicational hierarchy. Clin Linguist Phon. 1994; 8 (2): 91-107.

5. Howell J, Dean E. Treating Phonological Disorders in Children: Metaphon- Theory to practice. London: Whurr, 1994.

6. Williams AL. Multiple oppositions: theoretical foundations for an alternative contrastive intervention framework. Am J Speech-Lang Path. 2000a; 9:282-8.

7. Williams AL. A systematic perspective for assessment and intervention: A case study. Adv Speech-Lang Path. 2006; 8(3): 245-56.

8. Mota HB. Uma abordagem terapêutica baseada nos processos fonológicos no tratamento de crianças com desvios fonológicos [Dissertação]. Porto Alegre (RS): Pontifícia Universidade Católica do Rio Grande de Sul; 1990.

9. Keske-Soares M. Terapia fonoaudiológica fundamentada na hierarquia implicacional dos traços distintivos aplicada em crianças com desvios fonológicos [tese]. Porto Alegre (RS): Pontifícia Universidade Católica do Rio Grande de Sul; 2001. 10. Blanco AP. A generalização no modelo de ciclos modificado em pacientes com diferentes graus de severidade de desvio fonológico [dissertação]. Santa Maria (RS): Universidade Federal de Santa Maria; 2003.

11. Bagetti T, Mota HB, Keske-Soares M. Modelo de oposições máximas modificado: uma proposta de tratamento para o desvio fonológico. R. Soc. Bras. Fonoaudiol. 2005; 10 (1): 36-42.

12. Ardenghi LG, Mota HB, Keske-Soares M. A terapia Metaphon em casos de desvios fonológicos. Rev. Soc. Bras. Fonoaudiol. 2006; 11(2):106-15.

13. Ceron MI, Keske-Soares M. Terapia fonológica: a generalização a itens não utilizados no tratamento (outras palavras). Rev CEFAC. 2007; 9(4): 453-60.

14. Ceron MI, Keske-Soares M. Terapia fonológica: a generalização dentro de uma classe de sons e para outras classes de sons. Rev CEFAC. 2008; 10(3): 311-20.

15. Ceron MI, Keske-Soares M. Terapia fonológica: a generalização para outra posição na palavra. Ver. CEFAC. 2009; 11(2):199-206.

16. Mota HB, Keske-Soares M, Bagetti T, Ceron MI, Melo Filha, MGC. Análise comparativa da eficiência de três diferentes modelos de terapia fonológica. Pró-Fono. 2007; 19(1): 67-74.

17. Pagliarin KC, Ceron Ml, Keske-Soares M. Modelo de oposições múltiplas modificado: abordagem baseada em traços distintivos. Rev. soc. bras. fonoaudiol. 2009; 14 (3): 411-5.

18. Mota HB, Pereira LF. A generalização na terapia dos desvios fonológicos: experiência com duas crianças. Pró-Fono R. Atual. Cient. 2001; 13: 141-6. 19. Mota HB, Keske-Soares M, Ferla A, Zasso LV, Dutra LV. Estudo comparativo da generalização em três modelos de terapia para desvios fonológicos. Saúde: Revista do Centro de Ciência da Saúde. 2002;28(1-2):36-47 
20. Mota HB, Bagetti T, Keske-Soares M, Pereira LF. A generalização baseada nas relações implicacionais em sujeitos submetido à terapia fonológica. Pró-Fono R. Atual. Cient. 2005;17(1):99-110.

21. Barberena LS, Keske-Soares M, Mota H.B. Generalização no tratamento com o/R/ em um caso de desvio fonológico médio-moderado. Rev Soc Bras Fonoaudiol. 2004; 9: 229-36.

22. Pascoe M, Stackhouse J, Wells B. Phonological therapy within a psycholinguistic framework: Promoting change in a child with persisting speech difficulties. Int. J. Lang. Comm. Dis. 2005; 40 (2): 189-220.

23. Holm A, Crosbie S, Dodd B. Treating inconsistent speech disorders. In: Dodd D. Differential diagnosis and treatment of children with speech disorder. London: Whurr, 2005.

24. McIntosh B, Dodd B. Evaluation of Core Vocabulary intervention for treatment of inconsistent phonological disorder: Three treatment case studies. Child Lang. Teach. Ther. 2008; 25(1): 9-30.

25. Bowen C, Cupples L. Parents and children together (PACT): A collaborative approach to phonological therapy. Int. J. Lang. Comm. Dis. 1999; 34(1), 35-83.

26. Bowen C, Cupples L. PACT: Parents and children together in phonological therapy. Adv. Speech-Lang. Path. 2006; 8(3): 282-92.

27. Lancaster G, Keusch S, Levin A, Pring T, Martin S. Treating children with phonological problems: does an eclectic approach to therapy work?. Int. J. Lang. Comm. Dis. 2009; 29:1-12.

28. Hesketh A, Adams C, Nightingale C, Hall R. Phonological awareness therapy and articulatory training approaches for children with phonological disorders: a comparative outcome study. Int. J. Lang. Comm. Dis. 2000; 35 (3): 337-54.

29. Dodd B, Bradford A. A comparison of three therapy methods for children with different types of developmental phonological disorder. Int. J. Lang. Comm. Dis. 2000; 35 (2): 189-209.

30. Crosbie S, Holm A, Dodd B. Intervention for children with severe speech disorder: A comparison of two approaches. Int. J. Lang. Comm. Dis. 2005; 40 (4): 467-91.

31. Keske-Soares M, Brancalioni AR, Marini C, Pagliarin KC, Ceron Ml. Eficácia da terapia para desvios fonológicos com diferentes modelos terapêuticos. Pró-Fono R. Atual. Cient. 2008; 20 (3): 153-8.

32. Wren Y, Roulstone S. A comparison between computer and tabletop delivery of phonology therapy. Int. J. Speech-Lang. Path. 2008; 10(5): 346-63.
33. Dodd B, Crosbie S, Mclntosh B, Holm A, Harvey $C$, Liddy $M$, et al. The impact of selecting different contrasts in phonological therapy. Int. J. SpeechLang Path. 2008; 10(5): 334-45.

34. Pagliarin KC, Keske-Soares M, Mota HB. Terapia fonológica em irmãos com diferentes graus de gravidade do desvio fonológico. Rev. CEFAC. 2009; 11(1): 20-4.

35. Mota HB, Melo Filha MGC. Habilidades em consciência fonológica de sujeitos após realização de terapia fonológica. Pró-Fono R. Atual. Cient. 2009; 21 (2): 119-24.

36. Bernhardt B, Major E. Speech, language and literacy skills 3 years later: a follow-up study of early phonological and metaphonological intervention. Int. J. Lang. Comm. Dis. 2005; 40 (1): 1-27.

37. Denne M, Langdown N, Pring T, Roy P. Treating children with expressive phonological disorders: does phonological awareness therapy work in the clinic?. Int. J. Lang. Comm. Dis. 2005; 40 (4): 493-504.

38. Hesketh A, Dima E, Nelson. Teaching phoneme awareness to pre-literate children with speech disorder: a randomized controlled trial. Int. J. Lang. Comm. Dis. 2007; 42 (3): 251-71.

39. Spíndola RA, Payão LMC, Bandini HHM. Abordagem fonoaudiológica em desvios fonológicos fundamentada na hierarquia dos traços distintivos e na consciência fonológica. Rev CEFAC. 2007; 9(2): 180-9.

40. Mota HB. Pares mínimos: os contrastes fonológicos do Português Brasileiro. Pró-Fono. 2001; 13(1):98-106.

41. Mota HB, Keske-Soares M, Busanello AR, Balardin JB. Modificações no sistema fonológico provocadas por fonemas-alvo estimuláveis e não-estimuláveis. Rev. Soc. Bras. Fonoaudiol. 2006; 11(3):181-7.

42. Keske-Soares M, Mota HB, Pagliarin KC, Ceron MI. Estudo sobre os ambientes favoráveis à produção da líquida não-lateral /r/ no tratamento do desvio fonológico. Rev. soc. bras. fonoaudiol. 2007; 12(1): 48-54.

43. Gonçalves CS. Variáveis lingüísticas facilitadoras na reabilitação fonológica das líquidas não-laterais [Tese]. Porto Alegre (RS): Pontifícia Universidade Católica do Rio Grande de Sul; 2002.

44. Checalin MA. Estudo do Papel do Contexto Facilitador, segundo diferentes abordagens teóricas, na aquisição do r-fraco por crianças com desvio fonológico [Dissertação]. Santa Maria (RS): Universidade Federal de Santa Maria; 2008.

45. Blanco-Dutra AP. A aquisição das fricativas /f/, /v/, /S/ e /Z/ por crianças com desvio fonológico [Tese]. Porto Alegre (RS): Pontifícia Universidade Católica do Rio Grande do Sul; 2009. 
46. Keske-Soares M, Pagliarin KC, Ceron MI. Terapia fonológica considerando as variáveis linguísticas. Rev. soc. bras. fonoaudiol. 2009; 14 (2): 261-6.

47. Hodson BW. Identifying phonological patterns and projecting remediation cycles: Expediting intelligibility gains of a 7 year old Australian child. Adv. Speech-Lang. Path. 2006; 8(3): 257-64.

48. Keske-Soares M, Donicht G, Checalin MA, Ghisleni MRL. Generalização por 'reforço' ou 'contraste' no tratamento do desvio fonológico. Rev. Soc. Bras. Fonoaudiol. 2008; 13(4):391-7.

doi: $10.1590 / S 1516-18462011005000003$

RECEBIDO EM: 17/03/2010

ACEITO EM: 07/09/2010

Endereço para correspondência:

Fernanda Marafiga Wiethan

Rua Júlio Nogueira, $\mathrm{n}^{\circ} 130$ - Uglione

Santa Maria, RS

CEP: 97070-510

E-mail: fernanda_wiethan@yahoo.com.br 